\title{
Modeling the photochemical effects present during holographic grating formation in photopolymer materials
}

\author{
Michael R. Gleeson, John V. Kelly, Dusan Sabol, Ciara E. Close, Shui Liu, and \\ John T. Sheridan ${ }^{\mathrm{a}}$ \\ School of Electrical, Electronic, and Mechanical Engineering, College of Engineering, Mathematical, \\ and Physical Sciences, University College Dublin, Belfield, Dublin 4, Republic of Ireland
}

(Received 4 December 2006; accepted 30 April 2007; published online 26 July 2007)

\begin{abstract}
The development of a theoretical model of the processes present during the formation of a holographic grating in photopolymer materials is crucial in enabling further development of holographic applications. To achieve this, it is necessary to understand the photochemical and photophysical processes involved and to isolate their effects, enabling each to be modeled accurately. While photopolymer materials are practical materials for use as holographic recording media, understanding the recording mechanisms will allow their limitations for certain processes to be overcome. In this paper we report generalizations of the nonlocal polymer driven diffusion (NPDD) model to include the effects of photosensitive dye absorption and the inhibition effects. (C) 2007 American Institute of Physics. [DOI: 10.1063/1.2747536]
\end{abstract}

\section{INTRODUCTION}

The storage capabilities of photopolymer materials are under constant study due to their ability to record low loss, highly diffraction efficient volume holographic gratings. These self-processing materials are inexpensive and have performance characteristics that make them suitable for commercial use. To obtain the full potential of these materials requires quantitative insight into the processes present during recording. Developing accurate theoretical models, which are validated using reproducible experimental data sets, will allow crucial material parameters to be identified and controlled.

A study of the photochemical kinetics involved during holographic recording in our polyvinylalcohol/acrylamide (PVA/AA) based photopolymer ${ }^{1}$ material is presented here. Specifically, we aim to increase our understanding of what takes place inside the material during exposure, i.e., to explain the effects of the variation of the absorbance of the photosensitive dye with time $^{2-4}$ and the suppression of monomer radical production due to the presence of inhibitors. ${ }^{4-6}$ We thus aim to extend the validity of our nonlocal photopolymerization driven diffusion (NPDD) model $^{7-10}$ based on a deeper understanding of the photochemical and mass transport effects.

The paper is organized as follows: In Sec. II we begin by examining the rate equations of the photochemical processes present during grating formation and incorporate the suppression of radical production by inhibition effects. ${ }^{4}$ We also incorporate the effects of the change in the absorption of the material during exposure. A flow chart is presented, which succinctly summarizes the photochemical reactions, which take place during grating recording. In Sec. III we briefly review our material composition and method of preparation. In Sec. IV, having developed appropriate rate equations in

\footnotetext{
a) Author to whom correspondence should be addressed; FAX: +353-1-2830921; electronic mail: john.sheridan@ucd.ie
}

Sec. II, we derive and solve the resulting coupled equations numerically. ${ }^{7-10}$ In Sec. V simulations of the behavior of the monomer and polymer harmonics are presented. In Sec. VI comparisons between experimental data and the model are carried out. Fits are made and the results tend to support the assumptions made in deriving our equations. Estimated parameter values associated with inhibition and absorption are extracted and presented. A brief conclusion is given in Sec. VII.

\section{THE PHOTOCHEMICAL PROCESSES}

We begin by discussing radical chain polymerization with the assumption of bimolecular termination. ${ }^{11}$ In particular, we examine polymerization due to photoinitiation and examine the effect on excited dye molecules due to the presence of inhibitors such as oxygen. Furthermore, we include the effect of a time dependent transmittance, ${ }^{4,12}$ which describes the change in the material (dye) absorption during grating growth. A summary of our discussion in this section can be found in the flow chart in Fig. 1, which contains the equation numbers and references where appropriate.

Free radical polymerization involves three steps: initiation, propagation, and termination. The initiation step is considered to involve two primary reactions. The first is the production of free radicals by homolytic dissociation of an initiator or catalyst species $I$ to yield a pair of radicals $R^{*}$

$$
\stackrel{k_{d}}{\longrightarrow} 2 R^{*}
$$

where $k_{d}$ is the rate constant for the catalyst dissociation. The rate of this reaction is given by

$$
R_{d}=d\left[R^{*}\right] / d t=2 k_{d}[I],
$$

where $\left[R^{\bullet}\right]$ is the free radical concentration and $[I]$ is the initiator concentration. ${ }^{1,13}$ The second part of the initiation involves the addition of this radical to the first monomer molecule $M$ to produce the chain-initiation species $M_{1}^{*}$, 


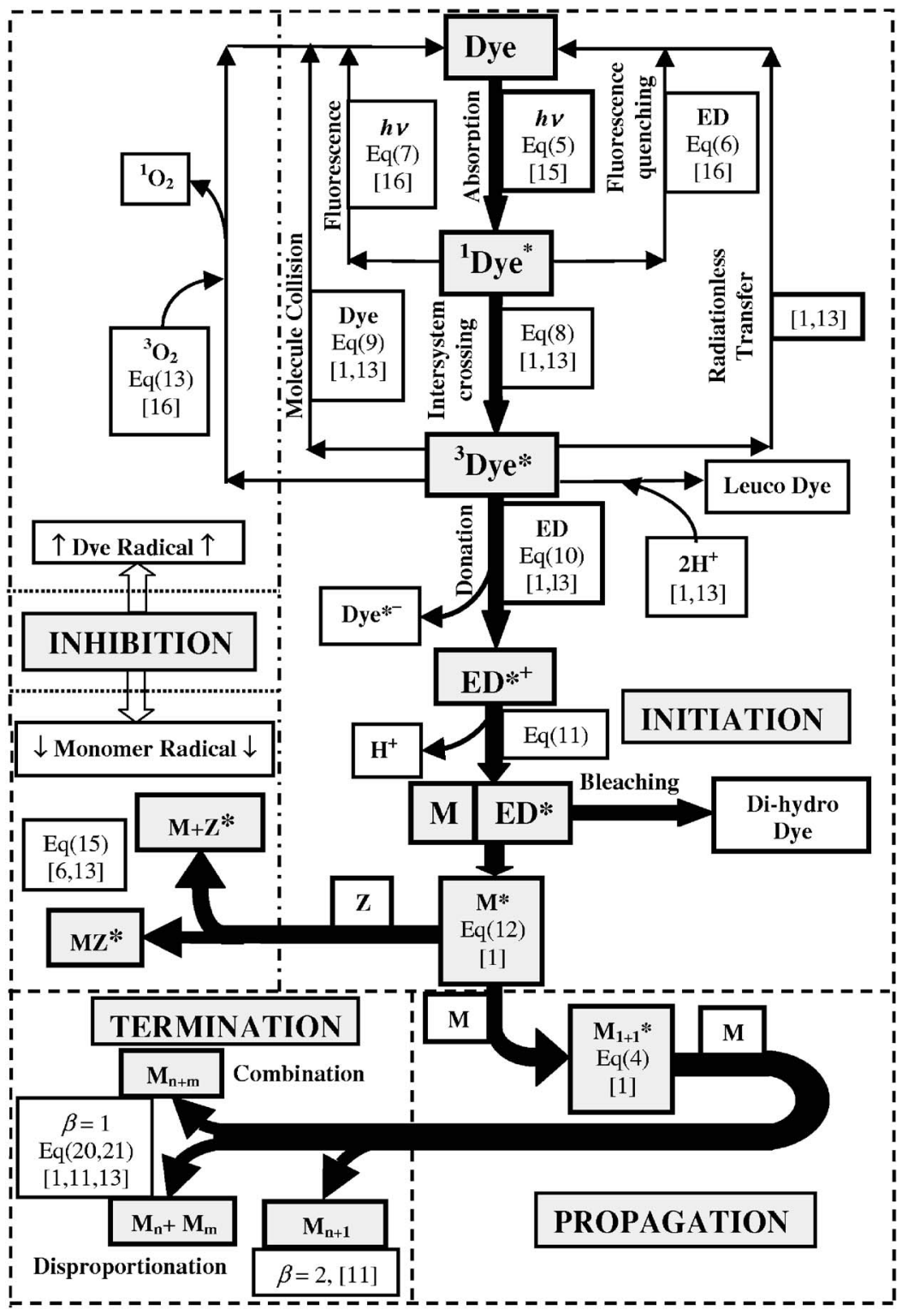

FIG. 1. Flow chart of the photochemical processes examined in Eqs. (5)-(21). Relevant references given in square brackets [-].

$$
R^{\cdot}+M \stackrel{k_{i}}{\longrightarrow} M_{1}
$$

where $k_{i}$ is the initiation rate constant. The radical $M_{1}^{*}$ then propagates by bonding with monomer molecules to form long polymer chains with an active tip known as a macroradical,

$$
M_{n}^{\cdot}+M \stackrel{k_{p}}{\longrightarrow} M_{n+1}^{\cdot},
$$

where $k_{p}$ is the propagation rate constant and $M_{n}^{\bullet}$ is a macroradical of $n$ monomeric units, where a monomeric unit is the largest constitutional unit contributed by a single monomer molecule. ${ }^{14}$

The initiator consists of a photosensitive dye (Dye) and a reducing agent. The dye can become excited in the presence of a photon and when excited can accept an electron from the reducing agent, i.e., a tertiary amine [triethanolamine, electron donor (ED)], ${ }^{15}$ and can then produce a free radical $R^{\circ}$.

When the dye molecule (Dye) is exposed to the light of a suitable wavelength, it absorbs a photon of light and is promoted to a singlet excited state, ${ }^{1}$ Dye ${ }^{*},{ }^{15}$

$$
\text { Dye }+h \nu \rightarrow{ }^{1} \mathrm{Dye}^{*}
$$

The singlet excited dye can return to the ground state by radiationless transfer to another molecule such as the ED. This process is known as fluorescence quenching, ${ }^{16}$ as can be seen in Fig. 1,

$$
{ }^{1} \mathrm{Dye}^{*}+\mathrm{ED} \rightarrow \text { Dye }+ \text { ED } .
$$

It can also revert back to the ground state Dye, by the emission of a photon by fluorescence ${ }^{16}$

$$
{ }^{1} \text { Dye }^{*} \rightarrow \text { Dye }+h \nu \text {. }
$$

The singlet state can also undergo intersystem crossing into the more stable and longer-lived triplet state (see Fig. 1), ${ }^{3}$ Dye, ${ }^{1,13}$ where

$$
{ }^{1} \text { Dye }^{*} \rightarrow{ }^{3} \text { Dye }^{*}
$$

This triplet state dye molecule can return to the ground state by radiationless transfer or by delayed emission of a photon. ${ }^{1,13}$ At high dye concentrations the triplet state dye 
molecules can be deactivated by collision with another dye molecule

$$
{ }^{3} \text { Dye }^{*}+\text { Dye } \rightarrow 2 \text { Dye. }
$$

The dye molecules can also undergo a reaction whereby it abstracts two hydrogen molecules from the electron donor to form the transparent (leuco) form of the dye. ${ }^{1,13}$

The actual production of free radicals takes place when the triplet state dye reacts with the electron donor. The electron donor donates an electron to the excited "triplet state" of the dye, leaving the dye with one unpaired electron and an overall negative charge,

$$
{ }^{3} \mathrm{Dye}^{*}+\mathrm{ED} \rightarrow \mathrm{Dye}^{\cdot-}+\mathrm{ED}^{\cdot+} .
$$

The electron donor radical cation then loses a proton and becomes a free radical

$$
\mathrm{ED}^{++} \rightarrow \mathrm{ED}^{*}+\mathrm{H}^{+} .
$$

The rate at which Eqs. (10) and (11) occur is $R_{d}$ in Eq. (2). When the acrylamide monomer is present, the free radical can undergo two different reactions. The first possible reaction is the initiation of the monomer radical $M_{1}^{*}$.

$$
\mathrm{ED}^{*}+M \rightarrow \mathrm{ED}-M_{1}^{*} \text {. }
$$

The second reaction the radical can undergo is dye bleaching. This occurs when the dye radical formed abstracts a hydrogen molecule from the electron donor free radical. An unstable electron donor intermediate and the transparent dihydro dye are formed in this reaction. The unstable intermediate then rearranges to form a more stable intermediate. Dye bleaching is an important process because it allows a grating to be fixed after recording. By bleaching any remaining dye no extra free radicals can be formed. This results in the grating being transparent. ${ }^{1,13}$

As can be seen in Fig. 1, when the photosensitive dye absorbs light it is promoted to the corresponding electronically excited states, the singlet excited state ${ }^{1}$ Dye or the triplet state ${ }^{3}$ Dye. Equation (13) shows that the longer life state, ${ }^{3}$ Dye, can be deactivated by the presence of initially dissolved ground state molecular oxygen ${ }^{3} \mathrm{O}_{2} \cdot{ }^{6}$

$$
{ }^{3} \text { Dye }^{*}+{ }^{3} \mathrm{O}_{2} \stackrel{k_{\rho}}{\longrightarrow} \text { Dye }+{ }^{1} \mathrm{O}_{2} .
$$

The rate at which this process occurs is $k_{\rho}$, and the rate of removal of the excited dye can be expressed as

$$
R_{\rho}=\frac{d\left[{ }^{1} \mathrm{O}_{2}\right]}{d t}=k_{\rho}\left[{ }^{3} \mathrm{Dye}^{*}\right]\left[{ }^{3} \mathrm{O}_{2}\right],
$$

where $R_{\rho}$ is the rate of removal of excited triplet state dye molecules by the dissolved oxygen in the material. This process causes a reduction in the amount of available excited dye molecules, which are responsible for the production of initiator radicals, which therefore causes an overall reduction in the rate of polymerization.

Moreover, the inhibiting ${ }^{3} \mathrm{O}_{2}$ can also react with the initiating primary radicals, formed through electron-proton transfer processes [see Eq. (10) and Fig. 1] and during the growth of macroradicals $M_{n}^{*}$, as in Eq. (4), which then give rise to inert products,

$$
M_{n}^{\cdot}+Z \stackrel{k_{z}}{\longrightarrow}\left(M_{n}+Z \text { and/or } M_{n} Z\right),
$$

where $Z$ is the inhibitor present, i.e., ${ }^{3} \mathrm{O}_{2}$ and $k_{z}$ is the rate constant for the inhibition process. ${ }^{13} Z$ acts either by adding to the propagating radical to form $M_{n} Z$ or by chain transfer of hydrogen or other radicals to yield $Z$ and the polymer chain $M_{n} \cdot{ }^{13}$ The rate of monomer radical scavenging $R_{z}$ can thus be expressed as

$$
R_{z}=\frac{d[Z]}{d t}=k_{z}[Z]\left[M_{n}^{*}\right] .
$$

The kinetics of this process are simplified if one assumes that $Z$ and $M_{n} Z$ do not reinitiate polymerization and also that they terminate without regeneration. All these possible oxygen-consuming reactions tend to suppress the creation of radicals and stop polymer chains from growing, therefore slowing the rate of polymerization resulting in an inhibition period. ${ }^{13}$ In this paper we assume that the effect of inhibition during the fabrication process is primarily due to the dissolved oxygen present in the material and that the inhibition period ends when all the dissolved oxygen has reacted.

The frequency of encounters between the free radicals is another factor determining the rate of polymerization. This can be accounted for using the cage effect. ${ }^{9,13}$ It is simply assumed that only some fraction $f$ of the free radicals produced react with monomer (in the starting reaction) because the PVA matrix physically restricts access.

From Eqs. (2), (10), (11), and (14) we see that the rate of production of free radicals, $R_{d}$, responsible for the production of monomer radicals in the presence of oxygen is given by

$$
R_{d}=f k_{d}[I]-R_{\rho},
$$

where $[I]$ is the initiator concentration. From Eq. (3) the rate of production of monomer radicals, $R_{i}$, can be written as

$$
R_{i}=d\left[M_{1}^{*}\right] / d t=k_{i}\left[R^{*}\right][M],
$$

where $[M]$ is the monomer concentration and $\left[R^{\circ}\right]$ is the primary radical concentration. In general, the rate of production of monomer radicals, $R_{i}$, is much greater than the rate of production of free radicals, $R_{d}$; therefore, initiator radicals are consumed as fast as they are generated. The fundamental rate-determining step is thus the decomposition of the initiator. Initiator radicals are consequently formed with a rate

$$
d\left[R^{*}\right] / d t=R_{d}-R_{i}=\left(f k_{d}[I]-R_{\rho}\right)-k_{i}\left[R^{*}\right][M]=0 .
$$

Thus, the rate of monomer radical generation, $R_{i}$, is equal to the chain-initiation rate and

$$
R_{i}=k_{i}\left[R^{*}\right][M]=f k_{d}[I]-R_{\rho} .
$$

Chain growth would continue in this way until the supply of monomer is exhausted were it not for the strong tendency of radicals to react in pairs to form paired-electron covalent bonds with a resulting loss of radical activity. At sufficiently low initiator concentrations, chain termination will occur mainly by combination, ${ }^{1,11,13}$

$$
M_{n}^{\cdot}+M_{m}^{\cdot} \stackrel{k_{t_{c}}}{\longrightarrow} M_{n+m},
$$

or by disproportionation, ${ }^{1,10,11,13}$ 


$$
M_{n}^{\cdot}+M_{m}^{\cdot} \stackrel{k_{t_{d}}}{\longrightarrow} M_{n}+M_{m}
$$

Based on these observations, the rate of termination $R_{t}$ can then be given by

$$
R_{t}=2 k_{t}\left[M^{\cdot}\right]^{2}
$$

where $k_{t}=k_{t_{c}}+k_{t_{d}}$ is the termination constant and $\left[M^{\circ}\right]$ is the total concentration of all chain radicals of size $M_{1}^{*}$ and larger. ${ }^{1,13}$

The steady state assumption for the radical concentration, applied to the combination of Eqs. (15b), (19), and (22), leads to

$$
\frac{d\left[M^{*}\right]}{d t}=R_{i}-2 k_{t}\left[M^{\bullet}\right]^{2}-k_{z}[Z]\left[M^{\bullet}\right]=0 .
$$

If it is assumed that much more monomers are consumed due to polymerization than are consumed in the initiation reaction, then the rate of polymerization from Eq. (3) is given as

$$
R_{p} \cong-d[M] / d t=k_{p}\left[M^{\bullet}\right][M],
$$

where $k_{p}$ is the propagation rate constant. As it is difficult to measure the concentration of monomer radicals, it is therefore desirable to eliminate $\left[M^{*}\right]$ from Eq. (23). Rearranging Eq. (24) and substituting $\left[M^{\circ}\right]$ into Eq. (23), we get ${ }^{13}$

$$
\frac{2 R_{p}^{2} k_{t}}{k_{p}^{2}[M]^{2}}+\frac{R_{p}[Z] k_{z}}{k_{p}[M]}-R_{i}=0 .
$$

Examining this equation it can be seen that $R_{p}$ is inversely proportional to the ratio of the rate constants for inhibition and propagation. This ratio is referred to as the inhibition constant, $z=k_{z} / k_{p}{ }^{13}$ It can be further seen that $R_{p}$ depends predominantly either on $R_{i}$ or on $\sqrt{R_{i}}$, depending on the relative magnitudes of the first two terms. Two limiting cases of this equation exist. When the second term is negligible compared to the first, the polymerization is not retarded and the equation simplifies to

$$
R_{p}=k_{p}[M]\left(\frac{R_{i}}{2 k_{t}}\right)^{1 / 2} .
$$

The second limiting case occurs when the retardation is strong, $k_{z} / k_{p} \gg 1$, and bimolecular termination ${ }^{1,11,13}$ is negligible. Under these conditions the first term in the equation can be neglected, and

$$
\frac{R_{p}[Z] k_{z}}{k_{p}[M]}-R_{i}=0
$$

Combining this with Eq. (24), we get

$$
R_{p}=\frac{k_{p}[M] R_{i}}{k_{z}[Z]}=-\frac{d[M]}{d t} .
$$

These equations show that the rate of retarded polymerization is proportional to the first power of the initiation rate. Furthermore, $R_{p}$ is inversely dependent on the inhibitor concentration. We have observed that the induction period of the inhibited polymerization is directly proportional to the concentration of the inhibitor. It is reasonable to assume that the

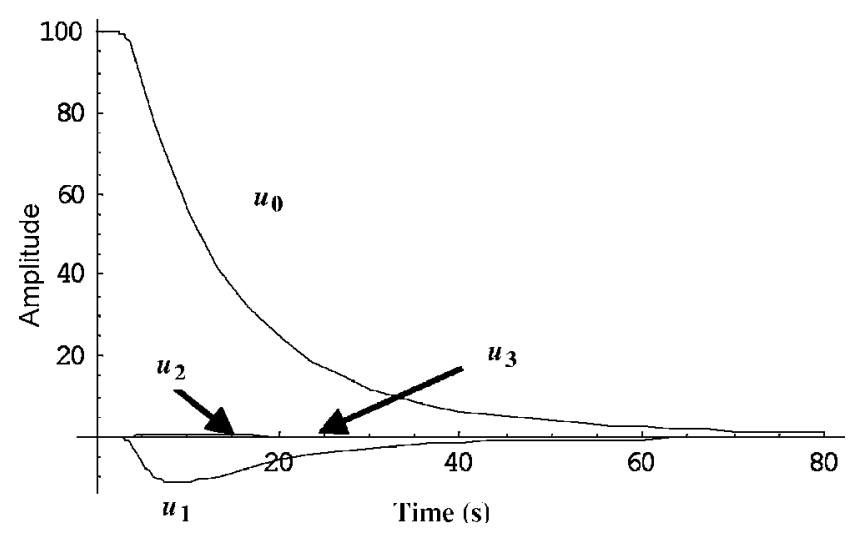

FIG. 2. First four Fourier harmonics of monomer concentration plotted as functions of exposure time.

rate of change of the inhibitor concentration $[Z]$ will decrease with time and be proportional to [Z],

$$
\frac{d[Z]}{d t} \propto-[Z]
$$

The constant of direct proportionality will be a function of the absorbed intensity $I_{a}$ and the number of radicals terminated per inhibitor molecule, $y$. If the initial concentration of inhibitor at $t=0$ is $\left[Z_{0}\right]$, then the solution of Eq. (29) can be written as

$$
[Z]=\left[Z_{0}\right] \exp \left[-c \frac{t}{t_{i}}\right] .
$$

$t_{i}$ is referred to as the inhibition time or the period of inhibition and is directly measured from the experimental growth curves (time until first nonzero diffraction efficiency measurement after the start of exposure). The $c$ parameter is estimated from fits to the experimental data. Some typical values chosen for these parameters are given in Sec. V for the simulations presented in Figs. 2 and 3. Solving the quadratic equation in Eq. (25) for $R_{p}$, and choosing the root which was physically reasonable give

$$
R_{p}=k_{p}[M] \frac{\sqrt{8 k_{t} R_{i}+k_{z}^{2}[Z]^{2}}-k_{z}[Z]}{4 k_{t}} .
$$

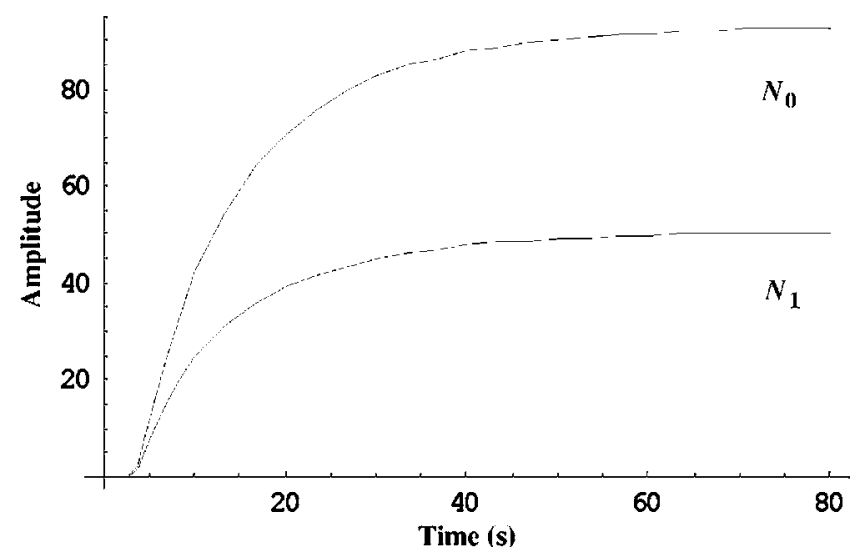

FIG. 3. First two Fourier harmonics of polymer concentration plotted as functions of exposure time. 
Examining the photochemical formation of free radicals, there are a number of initiation mechanisms, ${ }^{17}$ many involving a photochemical electron transfer reaction. If we reexamine the way in which the monomer radicals are formed, we see that they are dependent on the quantity of free radicals formed per photon absorbed and the intensity of the light used for recording. As the inhibitor present indirectly consumes some of the absorbed photons, there is a resulting reduction in the number of free radicals available for the initiation of monomer radicals and thus available for the formation of polymer chains. Therefore, the rate of initiation $R_{i}$ is given by

$$
R_{i}=f\left(\Phi^{\prime}-R_{\rho}^{\prime}\right) I_{a}(t),
$$

where $\Phi^{\prime}$ is the number of radicals produced per photon absorbed, $R_{\rho}^{\prime}$ is the number of radicals removed per photon absorbed due to the presence of inhibitor, and $I_{a}(t)$ is the intensity of light absorbed in moles of light quanta per $1 / \mathrm{s} .4,10,13$

We assume cosinusoidal spatially modulated illumination, $I(x)=I_{0}[1+V \cos (K x)],{ }^{18,19}$ where $V$ is the fringe visibility, $K=2 \pi / \Lambda$, the grating vector magnitude, and $\Lambda$ is the grating period. The concentration of photosensitizers, is related to the absorbed intensity by de Beer's law, ${ }^{13}$

$$
I_{a}(x, t)=I(x)\left\{1-\exp \left[-\varepsilon P_{s}(t) d\right]\right\}=I(x) A(t),
$$

where $\varepsilon$ is the molar absorptivity, $P_{s}(t)$ is the time dependent concentration of the photosensitizers (initiators), and $d$ is the photopolymer layer thickness. Since the concentration of photosensitizers is a function of time, the absorption of the layer, $A(t)$, also depends on time..$^{2-4,12}$ The inclusion of the absorption function $A(t)$ into the model enables an approximation of the time variation of the absorbed intensity. At the start of exposure the absorbance of the material will be high due to the large concentration of the photosensitive dye. This high absorption results in a large $I_{a}(x, t)$ and therefore a high rate of polymerization $R_{p}$. Similarly, as the concentration of photosensitive dye decreases, the absorbance of the layer can be assumed to decay exponentially, thus resulting in a lower rate of polymerization $R_{p}$.

As the absorbance of the material decreases, the amount of light that is transmitted through the material increases. ${ }^{4,12}$ Therefore, by measuring the material transmittance we can obtain information about the time varying absorbance of the material. We relate the absorbance $A(t)$ of the material to the transmittance $T(t)$ by ${ }^{4,12}$

$$
A(t)=1-B T(t),
$$

where $B$ is a loss fraction which is included to account for nonabsorptive loses, e.g., Fresnel boundary coefficients. Several transmittance curves were obtained for differing exposure intensities in our standard material layer. ${ }^{1,12,20}$ There is an initial nonzero transmittance at the beginning of the exposure as the material is never completely opaque. ${ }^{12}$ An approximate phenomenological expression for the time evolution of transmittance $T(t)$ was then determined based on fits to the experimental obtained data. $T(t)$ is estimated by fitting using the function
TABLE I. Composition of the acrylamide photopolymer material.

\begin{tabular}{ccc}
\hline \hline Component & Function & per $100 \mathrm{~cm}^{3}$ \\
\hline Polyvinylalcohol & Binder & $70 \mathrm{~cm}^{3}$ of $10 \%$ solution \\
Acrlyamide & Monomer & $2.4 \mathrm{~g}$ \\
Bisacrylamide & Cross-linker & $0.8 \mathrm{~g}$ \\
Erythrosin B & Dye & $16 \mathrm{~cm}^{3}$ of $\left(2 \times 10^{-4}\right) M$ \\
Triethanolamine & Electron donor & $8 \mathrm{~cm}^{3}$ \\
\hline \hline
\end{tabular}

$$
T(t)=E+G\left[1-\exp \left(-a_{0} t+a_{1} t^{2}\right)\right]
$$

where $E, G, a_{0}$, and $a_{1}$ are constant parameters related to the exposure intensity and the initial transmittance of the layer. ${ }^{4}$ Equation (35) is used in our simulations and data fitting procedures.

The polymerization rate $R_{p}$ in Eq. (31) can now be written as

$$
\begin{aligned}
R_{p} & =\frac{k_{p}[M]}{4 k_{t}}\left\{\sqrt{8 k_{t} f\left(\Phi^{\prime}-R_{\rho}^{\prime}\right) I_{a}(t)+k_{z}^{2}[Z]^{2}}-k_{z}[Z]\right\} \\
& =\frac{k_{p}[M][I(x)]^{\gamma}}{4 k_{t}}\left\{\sqrt{\left.8 k_{t} f\left(\Phi^{\prime}-R_{\rho}^{\prime}\right) A(t)+\frac{k_{z}^{2}[Z]^{2}}{I^{2 \gamma}(x)}-\frac{k_{z}[Z]}{I^{\gamma}(x)}\right\}}\right. \\
& =\kappa[M]\left[M^{\cdot}\right],
\end{aligned}
$$

where $\kappa$ is the polymerization rate constant.

In this section we have derived rate equations governing the photochemical processes involved in grating formation, which includes a term to explicitly account for radical suppression due to the effect of an inhibitor, i.e., oxygen. The time dependence for the absorbance of the photosensitive dye and photoinitiators is also included. ${ }^{3}$ In Fig. 1 a flow chart has been presented to succinctly summarize the photochemical processes.

\section{MATERIAL COMPOSITION AND PREPARATION}

Photopolymer materials can be made sensitive to a particular wavelength using a photosensitizing dye. The monomer used in this study is acrylamide, and a xanthene dye (erythrosin B) was used so that holographic recording could be carried out using a green $\lambda=532 \mathrm{~nm}$ solid-state crystal laser. We have also sensitized our acrylamide based material in the red (HeNe, $\lambda=633 \mathrm{~nm}$ ) using methylene blue, which is a thiazine dye.

Our PVA/AA material was made using the components listed in Table I. The material was prepared as follows:

(a) $10 \mathrm{~g}$ of PVA was added to $100 \mathrm{~cm}^{3}$ of de-ionized water and dissolved using a heater/stirrer. This solution is then allowed to cool, and $70 \mathrm{~cm}^{3}$ of this solution is transferred into a beaker.

(b) $8 \mathrm{~cm}^{3}$ of triethanolamine (ED) was added to the PVA solution and stirred thoroughly.

(c) $2.4 \mathrm{~g}$ of acrlyamide and $0.8 \mathrm{~g}$ of bisacrylamide were added to the PVA solution under a fume cupboard conditions and stirred until completely dissolved.

(d) For a green sensitive material $16 \mathrm{~cm}^{3}$ of $\left(2 \times 10^{-4}\right) M$ erythrosin B is added to the beaker. This step and subsequent steps were carried out under a safety light, as the 
material is now sensitive to green light. If we wish to sensitize in the red, then $16 \mathrm{~cm}^{3}$ of $\left(2 \times 10^{-4}\right) M$ methylene blue would have been added.

(e) The solution is then made up to $100 \mathrm{~cm}^{3}$ in a volumetric flask with de-ionized water.

(f) The solution is then stored in the dark ready for plate preparation.

To prepare dry material layers for holographic recording the solution prepared above is used as follows:

(1) The glass substrate on which the material is to be deposited (approximately $5 \times 4 \mathrm{~cm}^{2}$ ) is cleaned thoroughly using de-ionized water and acetone. Once cleaned the plates are placed on a level surface so that the photopolymer layers would adhere to the glass evenly, producing a layer of uniform thickness.

(2) $2 \mathrm{ml}$ of the photopolymer solution is then deposited evenly over the area of the glass plate using a syringe.

(3) Using this method, the typical material thickness is $100 \pm 10 \mu \mathrm{m}$. Different thicknesses can be obtained by depositing (drop casting) different quantities of material. The thickness and uniformity of these layers can be measured using a micrometer screw gauge.

(4) The plates are then left in the dark for approximately $24 \mathrm{~h}$ until dry. Drying times are dependent on the thickness of the material and the relative humidity.

(5) Once dry the plates are stored in a lightproof dessicator, ready for use.

\section{NONLOCAL POLYMERIZATION DRIVEN DIFFUSION MODEL}

In this section, extending our previous work, ${ }^{4}$ we incorporate the presence of inhibition and time varying absorption effects in the material into the NPDD model..$^{7-11}$

A dry photopolymer layer typically consists of a monomer, a binder, a cross-linker, an electron donor, and a photoinitiator. ${ }^{1,12,20}$ As the material is exposed to the recording beams, the monomer is polymerized, and the amount of polymerized monomer increases with exposure. In our material more monomer is polymerized in the bright fringes of the interference pattern than in the dark fringes. This results in a higher concentration of monomer in the dark regions than in the bright, and therefore a spatial monomer concentration gradient. The excess monomer will tend to diffuse into the bright regions. ${ }^{7-11,18}$

In Sec. II we derived the rate of polymerization. If $\gamma$ $=\frac{1}{2}$, then

$$
R_{p}=\kappa[I(x)]^{1 / 2} H[t][M],
$$

where $\quad H[t]=\left\{\sqrt{8 k_{t} f\left(\Phi^{\prime}-R_{\rho}\right) A(t)+k_{z}^{2}[Z]^{2} / I(x)}-k_{z}[Z] /\right.$ $\left.[I(x)]^{1 / 2}\right\} / 4 k_{t}$ takes into account the suppression of the production of free radicals, the scavenging of monomer radicals, and the change in the absorbance of the material with time. In this paper we assume that the main contributor to the observed inhibition effects arises due to the scavenging of the monomer radicals. The number of free radicals removed per photon absorbed due to the presence of an inhibitor, $R_{\rho}$, is therefore set to zero.

In standard notation the general one-dimensional (1D) NPDD equation governing the evolution of the monomer concentration distribution becomes ${ }^{7-9,11}$

$$
\begin{aligned}
\frac{\partial u(x, t)}{\partial t}= & \frac{\partial}{\partial x}\left[D(x, t) \frac{\partial u(x, t)}{\partial x}\right] \\
& -\int_{-\infty}^{+\infty} \int_{0}^{t} R\left(x, x^{\prime} ; t, t^{\prime}\right) F\left(x^{\prime}, t^{\prime}\right) \\
& \times\left[u\left(x^{\prime}, t^{\prime}\right)\right]^{\beta} d t^{\prime} d x^{\prime},
\end{aligned}
$$

where $u(x, t)$ is the free-monomer concentration, $D(x, t)$ is the monomer diffusion constant, $F(x, t)=F_{0}[1+V \cos (K x)]^{\gamma}$ is the polymerization rate, ${ }^{18,19}$ with $F_{0}=\kappa I_{0}^{\gamma}, R\left(x, x^{\prime} ; t, t^{\prime}\right)$ is the nonlocal material response function, and $\beta$ is the mode of termination. $^{7-9,11}$ The nonlocal response function represents the effect of initiation at location $x^{\prime}$ and time $t^{\prime}$ on the amount of monomer being polymerized at location $x$ and time $t .^{7-9,11}$ Assuming rapid chain growth, compared to other temporal effects, we can neglect the time nonlocality and let the nonlocal response function be $R\left(x, x^{\prime}\right),{ }^{7-9,11}$ in this way assuming an action at a distance in our response. We assume a Gaussian spatially nonlocal material response function

$$
R\left(x, x^{\prime}\right)=\frac{1}{\sqrt{2 \pi \sigma}} \exp \left[\frac{-\left(x-x^{\prime}\right)^{2}}{2 \sigma}\right],
$$

where $\sigma$ is the normalized nonlocal response parameter. ${ }^{7-9,11}$ The diffusion constant is defined by the expression $D(x, t)$ $=D_{0} \exp [-\alpha F(x) t]$, where $D_{0}$ is the initial diffusion constant and $\alpha$ is the diffusion coefficient decay parameter. ${ }^{7-9,11,18}$

The NPDD equations governing the bimolecular termination case $(\beta=1)$ have been derived elsewhere. ${ }^{7-9,11}$ Assuming that four harmonics provide a sufficiently accurate approximation, ${ }^{21}$ the monomer distribution can be well described by the following four harmonic, first-order coupled NPDD equations with $\xi=F_{0} t$ :

$$
\frac{d u_{0}(\xi)}{d t}=-f_{0} H(\xi) u_{0}(\xi)-\frac{1}{2} H(\xi)\left[f_{1} u_{1}(\xi)+f_{2} u_{2}(\xi)+f_{3} u_{3}(\xi)\right]
$$

$$
\begin{aligned}
\frac{d u_{1}(\xi)}{d t}= & -D K^{2} C h[\xi] u_{1}(\xi)-D K^{2} S h[\xi] u_{2}(\xi)-S_{1} H(\xi) \\
& \times\left[f_{1} u_{0}(\xi)+\left(f_{0}+\frac{f_{2}}{2}\right) u_{1}(\xi)+\frac{1}{2}\left(f_{1}+f_{3}\right) u_{2}(\xi)\right. \\
& \left.+\frac{1}{2}\left(f_{2}+f_{4}\right) u_{3}(\xi)\right], \\
\frac{d u_{2}(\xi)}{d t}= & -4 D K^{2} C h[\xi] u_{2}(\xi)+D K^{2} S h[\xi]\left[u_{1}(\xi)+3 u_{3}(\xi)\right] \\
& -S_{2} H(\xi)\left[f_{2} u_{0}(\xi)-\frac{1}{2}\left(f_{1}+f_{3}\right) u_{1}(\xi)\right. \\
& \left.+\left(f_{0}+\frac{f_{4}}{2}\right) u_{2}(\xi)+\frac{1}{2}\left(f_{1}+f_{5}\right) u_{3}(\xi)\right],
\end{aligned}
$$




$$
\begin{aligned}
\frac{d u_{3}(\xi)}{d t}= & -9 D K^{2} C h[\xi] u_{3}(\xi)+3 D K^{2} S h[\xi] u_{2}(\xi)-S_{3} H(\xi) \\
& \times\left[f_{3} u_{0}(\xi)-\frac{1}{2}\left(f_{2}+f_{4}\right) u_{1}(\xi)-\frac{1}{2}\left(f_{1}+f_{5}\right) u_{2}(\xi)\right. \\
& \left.-f_{0} u_{3}(\xi)\right],
\end{aligned}
$$

where $S_{i}=\exp \left(-i^{2} K^{2} \sigma / 2\right), u_{i}$ are the first four-monomer concentration Fourier harmonic amplitudes, $\mathrm{Ch}[\xi]=\exp$ $[-\alpha \xi(\sqrt{1-V}+\sqrt{1+V}) / 2] \cosh [\alpha \xi(\sqrt{1+V}-\sqrt{1-V}) / 2]$, and $\operatorname{Sh}[\xi]=\exp [-\alpha \xi(\sqrt{1-V}+\sqrt{1+V}) / 2] \sinh [\alpha \xi(\sqrt{1+V}-\sqrt{1-V})$ /2]. These first order coupled differential equations are solved numerically with the initial conditions $u_{0}(0)=100$ and $u_{m}(0)=0$ for all $m>0 . f_{0}, f_{1}, f_{2}$, and $f_{3}$ are the polymerization rate harmonic components, which depend on the fringe visibility $V$ and on $\gamma^{7-9,11,22}$ When $V=1$ and $\gamma=\frac{1}{2}, f_{0}$ $=2 \sqrt{2} / \pi$ and $f_{m}=(4 \sqrt{2} / \pi)\left[(-1)^{m+1} /\left(-1+4 m^{2}\right)\right]$.

The concentration of polymerized monomers after an exposure of duration $t$ is given by

$$
N(x, t)=\int_{0}^{t} \int_{-\infty}^{+\infty} R\left(x-x^{\prime}\right) F\left(x^{\prime}, t^{\prime}\right) u\left(x^{\prime}, t^{\prime}\right) d x^{\prime} d t^{\prime},
$$

yielding the following first two polymerization concentration spatial-harmonic components:

$$
\begin{aligned}
& N_{0}(\xi)=\int_{0}^{t} H\left(\xi^{\prime}\right)\left[f_{0} u_{0}\left(\xi^{\prime}\right)+\frac{f_{1}}{2} u_{1}\left(\xi^{\prime}\right)\right] d \xi^{\prime} \\
& N_{1}(\xi)=S_{1} \int_{0}^{t} H\left(\xi^{\prime}\right)\left[f_{1} u_{0}\left(\xi^{\prime}\right)+\left(f_{0}+\frac{f_{2}}{2}\right) u_{1}\left(\xi^{\prime}\right)\right] d \xi^{\prime}
\end{aligned}
$$

In numerically calculating the polymer harmonic concentrations, the higher order harmonics of monomer concentrations were assumed to be negligible (see Fig. 2). Again, as in the case of the monomer harmonics the polymer harmonic amplitudes are calculated numerically. The electromagnetic coupled wave theory ${ }^{23}$ predicts a relationship between the diffraction efficiency $\eta$ and the refractive index modulation $\Delta n$, of a thick sinusoidal transmission phase grating. If $N_{1}$ alone determines the first harmonic amplitude of the grating refractive index modulation, then $\Delta n(t)=C N_{1}(t)$ for some constant $C .{ }^{11}$ We note, however, that a much more complex relationship exists between the monomer and polymer concentrations and the various harmonic amplitudes $\Delta n_{i}{ }^{11,24}$

\section{SIMULATIONS}

Ultimately, we wish to apply our model to characterize our material behavior by fitting experimental growth curves. Before carrying out this procedure we wish to examine the general behavior predicted by our model.

Using Eq. (40), plots of the first four-monomer concentration harmonics were generated. The results are presented in Fig. 2, assuming an average exposure intensity of $I_{0}$ $=4 \mathrm{~mW} / \mathrm{cm}^{2}$. The following material parameter values were used to generate the graphs: $D=6 \times 10^{-11} \mathrm{~cm}^{2} / \mathrm{s}, \kappa$
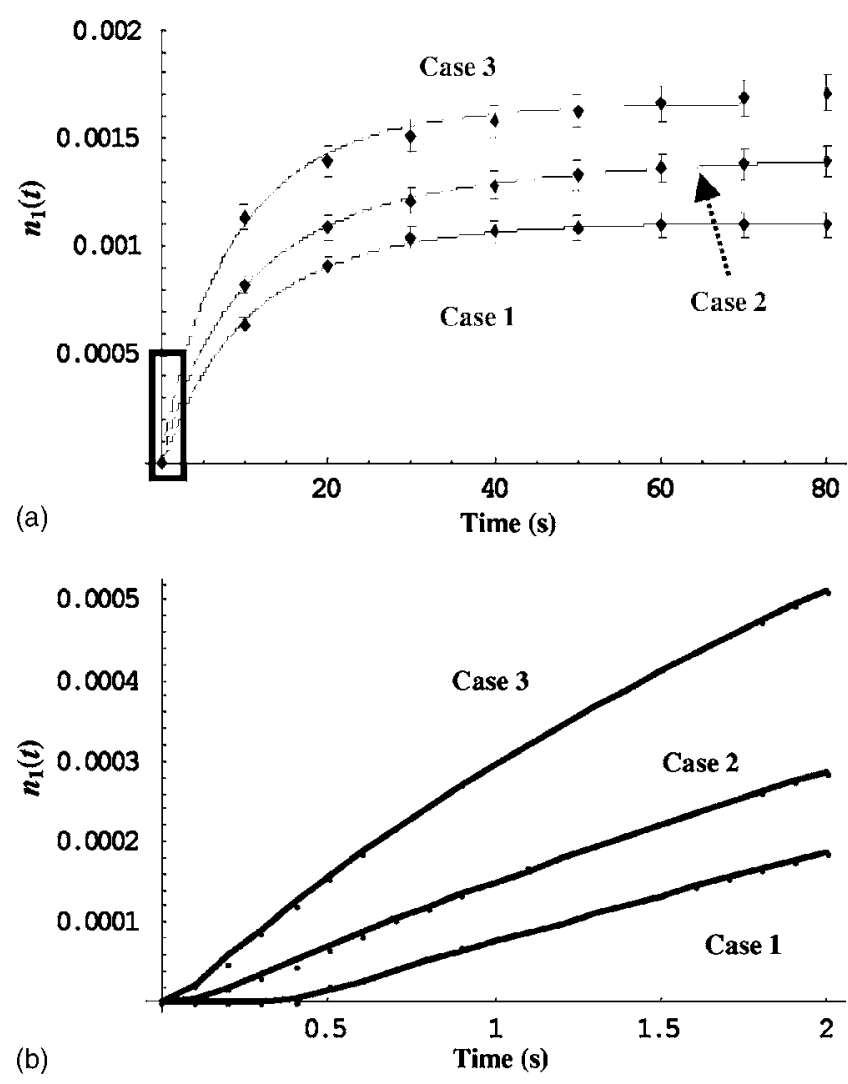

FIG. 4. (a) Fit to the experimentally obtained growth curves of refractive index modulation $( \pm 5 \%)$ vs exposure time for three different recording intensities: (1) $1.5 \mathrm{~mW} / \mathrm{cm}^{2}$, (2) $3.9 \mathrm{~mW} / \mathrm{cm}^{2}$, and (3) $4.8 \mathrm{~mW} / \mathrm{cm}^{2}$. (b) Initial subsection of (a) with fits to experimental data, illustrating the inhibition period.

$=0.06 \mathrm{~cm}^{2} \mathrm{~mW} \mathrm{~s}^{-1}, \quad C=3.1 \times 10^{-5} \mathrm{~cm}^{3} / \mathrm{mol}, \quad S=0.94, \quad \Phi^{\prime}$ $=0.07, \quad f=0.1, \quad V=1, \quad \gamma=\frac{1}{2}, \quad R_{\rho}^{\prime}=R_{\rho}=0, \quad \alpha=0, \quad k_{z}$ $=3000 \mathrm{~cm}^{2} \mathrm{~mW} \mathrm{~s}^{-1}$, and $k_{t}=0.004 \mathrm{~cm}^{2} \mathrm{~mW} \mathrm{~s}^{-1}$. A typical value for the loss fraction was empirically found to be $B$ $=0.9 \pm 0.05^{4}$

The absorbance function $A(t)$ is defined using Eq. (34) with the measured transmittance function constants [see Eq. (35)] estimated to be $E=0.176 \mathrm{~mW} / \mathrm{cm}^{2}, G=0.75 \mathrm{~mW} / \mathrm{cm}^{2}$, $a_{0}=1.92 \times 10^{-2} \mathrm{~s}^{-1}$, and $a_{1}=0.0347 \times 10^{-3} \mathrm{~s}^{-2}$.

The initial oxygen concentration present in the layer [Eq. (30) $]$ is assumed to be $\left[Z_{0}\right]=0.005 \mathrm{~g} / l,{ }^{4}$ and $c=2.5$. This estimated value for the concentration of dissolved oxygen appears reasonable relative to the densities and volumes of the other materials present. ${ }^{1,4}$ In Fig. 2 we note the presence of an initial dead band, $t_{\mathrm{i}} \sim 1.5 \mathrm{~s}$, caused by the inhibition process and the initiation period.

Figure 3 shows the corresponding predicted first two harmonics of the polymer concentration obtained using Eqs. (42) and (43). Once again, and in agreement with experiment, ${ }^{4,11}$ we note the existence of an inhibition period at the start of the exposure, and the gradual increase in the rate of polymerization during the transition between the inhibition period and steady state.

\section{EXPERIMENTAL RESULTS, NUMERICAL FITTING, AND PARAMETER ESTIMATION}

Previous studies examining the effects of inhibition ${ }^{4-6}$ have been carried out to determine the effects of exposure 
TABLE II. Directly measured and estimated parameter values. The transmittance function constants were produced from fits to the transmitted intensity of the two exposing beams measured during exposure.

\begin{tabular}{ccccccccc}
\hline \hline & $\begin{array}{c}I_{0} \\
\left(\mathrm{~mW} / \mathrm{cm}^{2}\right)\end{array}$ & $\begin{array}{c}d \\
(\mu \mathrm{m})\end{array}$ & $\Phi^{\prime}$ & $\begin{array}{c}k_{t} \\
\left(\mathrm{~cm}^{2} \mathrm{~mW} \mathrm{~s}\right)\end{array}$ & $\begin{array}{c}E \\
\left(\times 10^{-3}\right)\end{array}$ & $\begin{array}{c}G \\
\left(\mathrm{~mW}^{-1} \mathrm{~cm}^{2}\right)\end{array}$ & $\begin{array}{c}a_{0} \\
\left(\mathrm{~s}^{-1}\right)\end{array}$ & $\begin{array}{c}a_{1} \\
\left(\mathrm{~s}^{-2}\right) \\
\left(\times 10^{-2}\right)\end{array}$ \\
\hline Case 1 & 1.5 & 115 & 0.05 & 1.1 & 0.105 & 0.463 & 1.32 & 0.07 \\
Case 2 & 3.9 & 135 & 0.05 & 1.2 & 0.265 & 1.096 & 2.72 & 1.06 \\
Case 3 & 4.8 & 120 & 0.06 & 1.2 & 0.116 & 1.478 & 2.64 & 2.30 \\
\hline \hline
\end{tabular}

intensity changes, the effects of preexposure, and the effects of cover-plating (sealing). In the analysis presented in this paper we have modified the NPDD model to account for both the change in the absorption of the material with time and the presence of inhibition due to oxygen. We have also adjusted our previous inhibition model ${ }^{4,5}$ to include the process of monomer radical scavenging by the inhibitor. ${ }^{6}$

In Sec. V, simulations of the change in the monomer and polymer harmonics were generated using our model, illustrating the effect of the inhibition process and the change in the absorption of the material with exposure time. In this section we aim to increase the validity of our model by fitting experimentally obtained data and extracting key material parameters.

Using a standard holographic setup ${ }^{1,12,25}$ (exposing at $532 \mathrm{~nm}$ and probing at $633 \mathrm{~nm}$ ) for recording 1000 lines/mm unslanted transmission gratings, we obtained a set of growth curves for three different exposure intensities: (1) $1.5 \mathrm{~mW} / \mathrm{cm}^{2}$, (2) $3.9 \mathrm{~mW} / \mathrm{cm}^{2}$, and (3) $4.8 \mathrm{~mW} / \mathrm{cm}^{2}$. In each case the material was prepared in the manner described in Sec. III, with erythrosin B as the photosensitive dye. Numerical fits to the experimental data were performed using our adjusted four harmonic NPDD model (Sec. IV) and applying a numerical fitting algorithm. ${ }^{7-9} \mathrm{~A}$ comparison between the data and the resulting fits are presented in Figs. 4(a) and 4(b).

We fit the experimental data by first substituting "known" parameter values into our equations and then iteratively testing the fit quality over reasonable ranges of the unknown parameters. In this way estimates for $D, \kappa, S, R, t_{\mathrm{i}}$, and the constant $C$ are extracted. The chosen values used in the fitting algorithm were $\left[Z_{0}\right]=0.005 \mathrm{~g} / l, f=0.1, V=1, \gamma$ $=1 / 2, \alpha=0$, and $k_{z}=3 \times 10^{3} \mathrm{~cm}^{2} \mathrm{~mW} \mathrm{~s}^{-1}$. The characteristic parameters measured and those extracted from the fits to the experimental data presented in both Figs. 4(a) and 4(b) are given in Tables II and III.

In order to perform a stability analysis of our fitting pro- cedure, and also to provide error ranges to these estimated parameter values, we applied our fitting procedure to data sets artificially generated by adding and subtracting $5 \%$ of the measured value to the data points. In these artificial cases the mean squared error (MSE) values were significantly larger than for the data sets, and this is reflected in the ranges of MSE values presented in Table III [see the values in square [-] brackets].

Examining the estimates for $S, C$, and $\kappa$, it can be seen that they are comparable to those presented in the literature. ${ }^{4,9,11}$ Furthermore, the values obtained for the monomer diffusion rate, $D$, lie within the range of the estimations provided by our current studies on diffusion rates in photopolymers. ${ }^{26}$ The values of the inhibition period $t_{i}$ follow the trend predicted in previous studies. ${ }^{4}$ Low MSE values are obtained, which is evident in the quality of the fits presented in Figs 4(a) and 4(b). In this analysis bimolecular termination is assumed $(\beta=1)$. However, recently, we have indicated that better fits can be achieved when a primary termination mechanism $(\beta=2)$ is assumed. ${ }^{9}$ It is possible that both mechanisms of termination are present during grating growth, and this could explain the reduction in fit quality seen in the higher exposure intensity case (see case 3 ). The values obtained for the bulk parameter $R$ are also reasonable. ${ }^{4,9,11,26}$

Figure 4(b) shows the initial subsection of the experimental growth curves, as outlined in Fig. 4(a). Applying the same fitting procedure as used in fitting Fig. 4(a), almost identical parameter values were extracted. Thus, we have demonstrated our ability to accurately predict the behavior of the photopolymers at the early stages of exposure during the transition from inhibition to polymerization (with MSE fitting values of the order of $10^{-11}$ ).

\section{CONCLUSIONS}

Starting with a detailed description of the photochemical processes taking place in a PVA/AA based photopolymer

TABLE III. Characteristic parameters estimated using numerical fitting to experimental data.

\begin{tabular}{|c|c|c|c|c|c|c|c|}
\hline & $\begin{array}{l}t_{i} \\
(\mathrm{~s})\end{array}$ & $\begin{array}{c}D \\
\left(\mathrm{~cm}^{2} / \mathrm{s}\right) \\
\left(\times 10^{-11}\right)\end{array}$ & $\begin{array}{c}C \\
\left(\mathrm{~cm}^{3} / \mathrm{mol}\right) \\
\left(\times 10^{-5}\right)\end{array}$ & $\begin{array}{c}\kappa \\
\left(\mathrm{cm}^{2} \mathrm{~mW} \mathrm{~s}^{-1}\right)\end{array}$ & $S$ & $R$ & $\begin{array}{c}\text { MSE } \\
\left(\times 10^{-10}\right)\end{array}$ \\
\hline Case 1 & 0.4 & $\begin{array}{c}8.33 \\
\pm 0.41\end{array}$ & $\begin{array}{c}2.07 \\
\pm 0.88\end{array}$ & $\begin{array}{c}0.058 \\
\pm 0.004\end{array}$ & $\begin{array}{c}0.94 \\
\pm 0.01\end{array}$ & $\begin{array}{l}4.62 \\
\pm 0.5\end{array}$ & $\begin{array}{l}0.87 \\
{[4.6]}\end{array}$ \\
\hline Case 2 & 0.2 & $\begin{array}{c}6.02 \\
\pm 1.92\end{array}$ & $\begin{array}{c}2.67 \\
\pm 0.24\end{array}$ & $\begin{array}{c}0.057 \\
\pm 0.004\end{array}$ & $\begin{array}{c}0.95 \\
\pm 0.01\end{array}$ & $\begin{array}{l}2.10 \\
\pm 0.6\end{array}$ & $\begin{array}{l}0.79 \\
{[4.9]}\end{array}$ \\
\hline Case 3 & 0.1 & $\begin{array}{c}6.01 \\
\pm 0.85\end{array}$ & $\begin{array}{l}3.40 \\
\pm 0.1\end{array}$ & $\begin{array}{c}0.066 \\
\pm 0.006\end{array}$ & $\begin{array}{c}0.94 \\
\pm 0.01\end{array}$ & $\begin{array}{l}1.62 \\
\pm 0.3\end{array}$ & $\begin{array}{l}1.23 \\
{[6.2]}\end{array}$ \\
\hline
\end{tabular}


material, we have developed a NPDD model that includes the effects of oxygen based inhibition and bleaching of the photosensitive dye during exposure.

It was previously assumed that the main process responsible for the presence of inhibition in our photopolymer material was the removal of excited triplet state dye molecules. ${ }^{4,5}$ This removal of dye ultimately causes a suppression of the production of free radicals and stopping polymerization. In this paper it has been shown that a reaction between the inhibitor and the monomer radicals also results in a consistent stopping of chain growth, producing an equivalent retardation of the polymerization process. While it is possible that some combination of these two effects is present during grating formation it was assumed in this paper that the reaction between inhibitor and monomer radicals was dominant, i.e., it was assumed that $R_{\rho}=0$.

Previously, we have demonstrated that it is possible to reduce the inhibition period $t_{i}$ significantly by (a) increasing the intensity of the holographic exposing beams and (b) by nonholographic preexposure combined with layer coverplating (sealing). ${ }^{4}$ Another proposal is to eliminate $t_{i}$ through the addition of a second photosensitive dye and a suitable preexposure. ${ }^{6}$ Any dissolved oxygen present within the material will act to suppress the resulting excited state dye molecules. During any subsequent holographic exposure the primary photosensitizing dye is unaffected and remains ready for recording at the appropriate wavelength. We note that rose bengal and methylene blue are among the most efficient ${ }^{1} \mathrm{O}_{2}$ generators. ${ }^{6}$

The NPDD model presented in this paper suffers from several serious deficiencies that limit the accuracy of the results. Neither the inhibition nor the initiation models proposed have been derived from first principles. Furthermore, the discussion neglects all three-dimensional effects, i.e., variations with depth. We note that previously a more exact expression for the refractive index modulation evolution calculated using the Lorentz-Lorenz equation has been applied. ${ }^{11}$ Furthermore, the numerical accuracy of the reported simulations is limited by our inclusion of only four monomer harmonics in our calculations. ${ }^{21}$ To avoid such numerical difficulties a fully rigorous finite time domain analysis has been used. ${ }^{10,11}$ Other effects such as the choice of the dominant chain termination mechanisms and material shrinkage ${ }^{3,11,27,28}$ must also be included to enable an accurate and physical model to emerge.

This study is clearly important and topical, as both inhibition and dye bleaching have been observed in a wide range of materials. ${ }^{29}$ They must be understood in order to determine material applicability in a wide range of areas such as holographic data storage, ${ }^{30,31}$ photoembossing,${ }^{27}$ and diffractive optical element fabrication. ${ }^{32-34}$

\section{ACKNOWLEDGMENTS}

We acknowledge the support of Enterprise Ireland and Science Foundation Ireland through the Research Innovation and Proof of Concept Funds and the Basic Research and Research Frontiers Programs. We would also like to thank the Irish Research Council for Science, Engineering, and Technology and The International Society for Optical Engineering SPIE for a SPIE Educational Scholarship.

${ }^{1}$ J. R. Lawrence, F. T. O’Neill, and J. T. Sheridan, Optik (Stuttgart) 112, 449 (2001).

${ }^{2}$ S. Gallego, M. Ortuno, C. Neipp, and J. T. Sheridan, Opt. Express 13, 3543 (2005)

${ }^{3}$ S. Blaya, L. Carretero, R. F. Madrigal, M. Ulibarrena, P. Acebal, and A. Fimia, Appl. Phys. B: Lasers Opt. B77, 639 (2003).

${ }^{4}$ M. R. Gleeson, J. V. Kelly, C. E. Close, F. T. O'Neill, and J. T. Sheridan, J. Opt. Soc. Am. B 23, 2079 (2006)

${ }^{5}$ A. V. Galstyan, R. S. Hakobyan, and S. H. T. Galstian, http://e-lc.org/ Documents/T.V Galstian_2004_05_05_11_13_17.pdf

${ }^{6}$ A. Fimia, N. Lopez, F. Mateos, R. Sastre, J. Pineda, and F. Amat-Guerri, J. Mod. Opt. 40, 699 (1993).

${ }^{7}$ J. T. Sheridan and J. R. Lawrence, J. Opt. Soc. Am. A 17, 1108 (2000).

${ }^{8}$ J. R. Lawrence, F. T. O'Neill, and J. T. Sheridan, J. Opt. Soc. Am. B 19, 621 (2002)

${ }^{9}$ J. V. Kelly, F. T. O’Neill, and J. T. Sheridan, J. Opt. Soc. Am. B 22, 407 (2005).

${ }^{10}$ S. Wu and E. N. Glytsis, J. Opt. Soc. Am. B 20, 1177 (2003).

${ }^{11}$ J. V. Kelly, M. R. Gleeson, C. E. Close, F. T. O’Neill, J. T. Sheridan, S. Gallego, and C. Neipp, Opt. Express 13, 6990 (2005).

${ }^{12}$ M. R. Gleeson, J. V. Kelly, F. T. O'Neill, and J. T. Sheridan, Appl. Opt. 44, 1 (2005).

${ }^{13}$ G. Odian, Principles of Polymerization (Wiley, New York, 1991).

${ }^{14}$ IUPAC, Compendium of Chemical Terminology 2nd ed. (Oxford, Blackwell Science, 1997).

${ }^{15}$ J. B. Birk, Organic Molecular Photophysics (Wiley, New York, 1975), Vol. 2.

${ }^{16}$ A. Gilbert and J. Baggott, Essentials of Molecular Photochemistry (Blackwell Scientific, Oxford 1991).

${ }^{17}$ W. J. Tomlinson, Advances in Photochemistry (Wiley, New York, 1980).

${ }^{18}$ G. Zhao and P. Mouroulis, J. Mod. Opt. 41, 1929 (1994).

${ }^{19}$ J. H. Kwon, H. C. Hwang, and K. C. Woo, J. Opt. Soc. Am. B 16, 1651 (1999).

${ }^{20}$ F. T. O'Neill, J. R. Lawrence, and J. T. Sheridan, Opt. Eng. (Bellingham) 40, 533 (2001).

${ }^{21}$ J. T. Sheridan, J. V. Kelly, G. O'Brien, M. R. Gleeson, and F. T. O’Neill, J. Opt. A, Pure Appl. Opt. 6, 1089 (2004).

${ }^{22}$ J. T. Sheridan, M. R. Gleeson, C. E. Close, and J. V. Kelly, J. Nanosci. Nanotechnol. 7, 232 (2007).

${ }^{23}$ H. Kogelnik, Bell Syst. Tech. J. 48, 2909 (1969).

${ }^{24}$ I. Aubrecht, M. Miller, and I. Koudela, J. Mod. Opt. 45, 1465 (1998).

${ }^{25}$ F. T. O'Neill, J. R. Lawrence, and J. T. Sheridan, Optik (Stuttgart) 111, 459 (2000).

${ }^{26}$ C. E. Close, M. R. Gleeson, F. T. O'Neill, J. V. Kelly, and J. T. Sheridan, Proc. SPIE 5827, 346 (2005).

${ }^{27}$ F. T. O'Neill, A. J. Carr, S. M. Daniels, M. R. Gleeson, J. V. Kelly, J. R. Lawrence, and J. T. Sheridan, J. Mater. Sci. 40, 4129 (2005)

${ }^{28}$ F. T. O'Neill, J. R. Lawrence, and J. T. Sheridan, Opt. Eng. (Bellingham) 40, 533 (2001)

${ }^{29}$ R. K. Kostuk, Appl. Opt. 38, 1357 (1999).

${ }^{30}$ Holographic Data Storage, Springer Series in Optical Sciences, edited by H. J. Coufal, D. Psaltis, and G. T. Sincerbox (Springer, Berlin, 2000).

${ }^{31}$ J. T. Sheridan, M. R. Gleeson, J. V. Kelly, and F. T. O'Neill, Opt. Lett. 30, 239 (2005).

${ }^{32}$ H. Kobolla, J. T. Sheridan, E. Gluch, J. Schmidt, R. Völkel, J. Schwider, and N. Streibl, J. Mod. Opt. 40, 613 (1993).

${ }^{33}$ H. Kobolla, J. Schmidt, J. T. Sheridan, N. Streibl, and R. Völkel, J. Mod. Opt. 39, 881 (1992).

${ }^{34}$ J. Schmidt, R. Völkel, W. Stork, J. T. Sheridan, J. Schwider, N. Streibl, and F. Durst, "Diffractive beamsplitter for laser doppler velocimetry," Opt. Lett. 17, 1240 (1992). 